\title{
Duration and exclusiveness of breastfeeding and childhood asthma-related symptoms
}

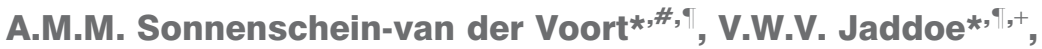 \\ R.J.P. van der Valk*,\#, , S.P. Willemsen ${ }^{\S}$, A. Hofman ", H.A. MolI', \\ J.C. de Jongste ${ }^{\#}$ and L. Duijts*,\#,
}

ABSTRACT: The aim of our study was to examine the associations of breastfeeding duration and exclusiveness with the risks of asthma-related symptoms in preschool children, and to explore whether these associations are explained by atopic or infectious mechanisms.

This study was embedded in a population-based prospective cohort study of 5,368 children. Information on breastfeeding duration, exclusiveness and asthma-related symptoms, including wheezing, shortness of breath, dry cough and persistent phlegm, was obtained by questionnaires.

Compared with children who were breastfed for 6 months, those who were never breastfed had overall increased risks of wheezing, shortness of breath, dry cough and persistent phlegm during the first 4 yrs (OR 1.44 (95\% Cl 1.24-1.66), 1.26 (1.07-1.48), 1.25 (1.08-1.44) and 1.57 (1.29-1.91), respectively). Similar associations were observed for exclusive breastfeeding. The strongest associations per symptom per year were observed for wheezing at 1 and 2 yrs. Additionally adjusted analyses showed that the associations of breastfeeding with asthma-related symptoms were not explained by eczema but partly by lower respiratory tract infections.

Shorter duration and nonexclusivity of breastfeeding were associated with increased risks of asthma-related symptoms in preschool children. These associations seemed, at least partly, to be explained by infectious, but not by atopic, mechanisms.

KEYWORDS: Asthma, duration of breastfeeding, exclusive breastfeeding, wheezing

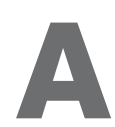

sthma-related symptoms are common in early childhood and are a leading cause of morbidity [1]. Known risk factors in early life for asthma-related symptoms include birth weight, gestational age, parental socioeconomic status, ethnicity, presence of siblings, daycare attendance, family history of asthma or atopy and parental smoking [2]. A substantial body of evidence suggests that breastfeeding is also associated with a reduced risk of childhood asthma and asthmarelated symptoms [3-14]. Some studies have reported stronger protective effects of breastfeeding on asthma in children with a positive family history of asthma or allergy $[8,15,16]$, whereas others did not $[6,11,12]$. Studies that focused on asthma later in life showed inconsistent results $[5,7,8,10,11]$. Breastfeeding might affect the risk of childhood asthma because of a mediating effect of atopy, infections or both. Underlying mechanisms might include immunoglobulin A, cytokines, especially transforming growth factor- $\beta 1$, and long-chain fatty acids in breast milk that stimulate the infant's immune system [17]. Furthermore, glycans help the innate immune system to inhibit pathogen binding to the host cell target ligand [18], and changes in the delicate balance between pro- and antiinflammatory compounds [19]. Various methodological issues might have influenced results from previous studies. These include recall bias of feeding habits in retrospective studies, differences in information about duration and exclusiveness of breastfeeding, and adjustment for confounders $[2,5-7,11,14]$.

Therefore, in a population-based prospective cohort study, we examined the associations of the duration and exclusiveness of breastfeeding with the risks of asthma-related symptoms during the

\section{AFFILIATIONS}

*The Generation R Study Group, Depts of \#Paediatrics, Division of Respiratory Medicine,

"Epidemiology,

+Paediatrics, and

${ }^{\S}$ Biostatistics, Erasmus Medical Center, Rotterdam, The Netherlands.

CORRESPONDENCE

L. Duijts

The Generation R Study Group (room Ae 012)

Erasmus Medical Center

P0 Box 2040

3000 CA Rotterdam

The Netherlands

E-mail: I.duijts@erasmusmc.nl

Received:

Nov 182010

Accepted after revision: June 172011

First published online:

July 202011 
first 4 yrs, and examined whether any association is explained by atopic or infectious mechanisms.

\section{METHODS}

\section{Design and cohort}

This study was embedded in the Generation R Study, a population-based prospective cohort study of pregnant females and their children from fetal life onwards in Rotterdam, the Netherlands, and has previously been described in detail [20]. The study protocol was approved by the Medical Ethical Committee of the Erasmus Medical Centre, Rotterdam. Written informed consent was obtained from all participants. In total, 7,295 children and their parents participated in the post-natal phase of the study. From those children, twins $(n=179)$ and second or third children of the same mother in the study $(n=539)$ were excluded from the present analyses to prevent bias due to correlation (online supplementary material fig. S1). Of the remaining children, breastfeeding and asthma-related symptom data were available of 5,368 children.

\section{Breastfeeding duration and exclusiveness}

Information about breastfeeding initiation and continuation was obtained by postal questionnaires at the ages of 2,6 and 12 months after birth [20]. The duration of breastfeeding was assessed by asking whether they ever breastfed their child (no or yes) and at what age (weeks) they stopped breastfeeding. Subsequently, breastfeeding duration was categorised into four groups: never; $<3$ months; $3-6$ months and $\geqslant 6$ months. Exclusive breastfeeding was defined using information on the introduction of other milk or solids. The information about exclusiveness of breastfeeding was combined and categorised into the following three breastfeeding categories: never; nonexclusive breastfeeding until 4 months, and exclusive breastfeeding until 4 months.

\section{Asthma-related symptoms}

Information on asthma-related symptoms was obtained by questionnaires at the ages of 1, 2, 3 and 4 yrs. Questions about asthma-related symptoms were adapted from the International Study on Asthma and Allergy in Childhood (ISAAC) [21]. Response rates for these questionnaires were 71, 76, 72 and 73\%, respectively. Information about asthma-related symptoms in the past year included wheezing (never, 1-3 times and $>4$ times), shortness of breath (never, 1-3 times and $>4$ times), dry cough at night (no or yes) and mucus congestion (no or yes). Parents also reported information about doctor-attended eczema and lower respiratory tract infections (pertussis, bronchitis, bronchiolitis or pneumonia) in the past year, and this information was used as a marker of atopy and infection, respectively.

\section{Covariates}

Information on parental history of asthma or atopy, socioeconomic status, ethnicity, parity and pet keeping were obtained by questionnaire, completed by the mother at enrolment. Information about active maternal smoking was obtained by postal questionnaires sent during the first, second and third trimester of pregnancy and combined into smoking (no or yes) [20]. Socioeconomic status was assessed using the highest educational level achieved by the parents. Maternal ethnicity was based on country of birth of both the mother and her parents [20]. We used parity as a proxy for siblings, the correlation between those variables was good $(\kappa=0.894)$. Birth weight, gestational age and sex of the children were obtained from midwife and hospital registries at birth. Home-sent questionnaires at the ages of 6 and 12 months provided information about daycare attendance.

\section{Data analysis}

Longitudinal analyses

We used generalised estimating equations (GEEs) to examine the longitudinal effects of duration and exclusiveness of breastfeeding with each asthma-related symptom (no or yes) from the ages of 1 to 4 yrs. With GEE analyses, repeatedly measured asthma-related symptoms can be analysed over time, taking into account that these repeated measurements are correlated within the same subject. Also, breastfeeding and age might be correlated and, therefore, breastfeeding was used in the model as a timedependent variable. Covariates were not repeatedly measured over time and were introduced in the models as time independent.

\section{Additional confounder analyses}

To assess whether the associations of breastfeeding with asthmarelated symptoms could be explained by atopic or infectious mechanisms, we additionally adjusted the analyses for doctorattended eczema and lower respiratory tract infections measured at the corresponding ages.

\section{Effect modification analyses}

To assess the potential modifying effect of parental history of asthma or atopy, we added parental history of asthma or atopy (no or yes) as an interaction term with exclusive breastfeeding in the GEE models, with wheezing as the outcome (wheezing= exclusivity of breastfeeding+parental history of asthma or atopy+ exclusivity of breastfeeding $\times$ parental history of asthma or atopy +other confounders). Thereafter, we stratified our GEE models for breastfeeding exclusivity by parental history of asthma or atopy.

\section{Survival analysis}

We performed a discrete survival analysis to calculate time to first asthma-related symptom according to breastfeeding duration and exclusiveness. For these analyses, the four different asthmarelated symptoms were combined into one categorical variable, asthma-related symptom (no or yes).

\section{Dose-response analysis}

The associations of breastfeeding duration and exclusivity with frequencies of asthma-related symptoms at the ages of 1, 2, 3 and 4 yrs were analysed using multiple logistic regression analysis.

Missing data in the covariates were imputed using the multiple imputation procedure, which is used to select possible values for a missing response. Five imputed data sets were created and analysed together. All models were adjusted for potential confounders, including parental age, education, ethnicity, smoking habits, maternal parity, children's sex, gestational age, birth weight, parental history of asthma or atopy, daycare attendance and pet keeping. Tests for trends were performed by including the breastfeeding categories as continuous variables in the regression models. All measures of association are presented with their 95\% confidence intervals (CIs). The statistical analyses were performed using SPSS version 17.0 for Windows (SPSS Inc., Chicago, IL, USA) and SAS 9.2 (SAS Institute, Cary, NC, USA). 
TABLE 1 Characteristics of children and their parents according to breastfeeding duration

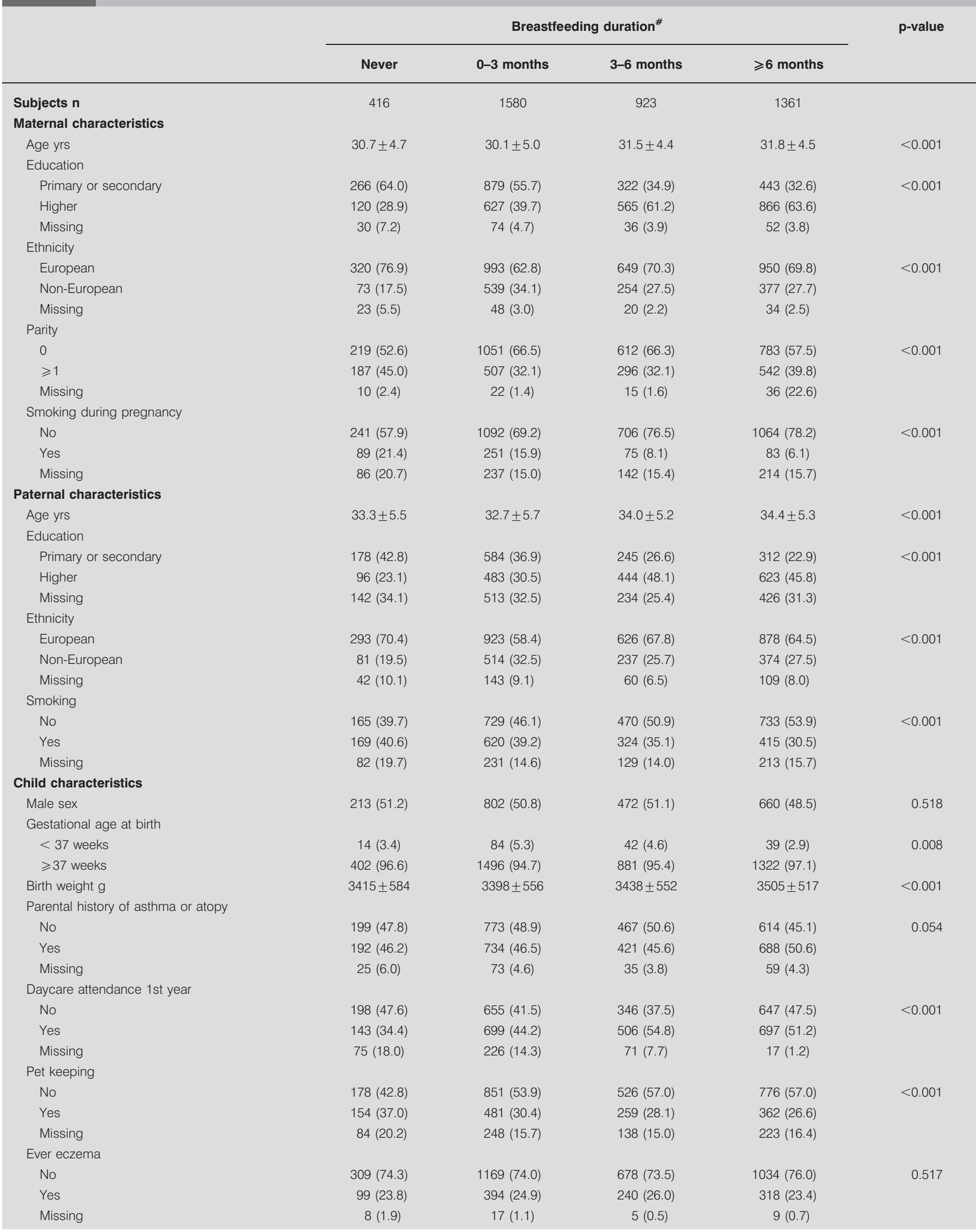




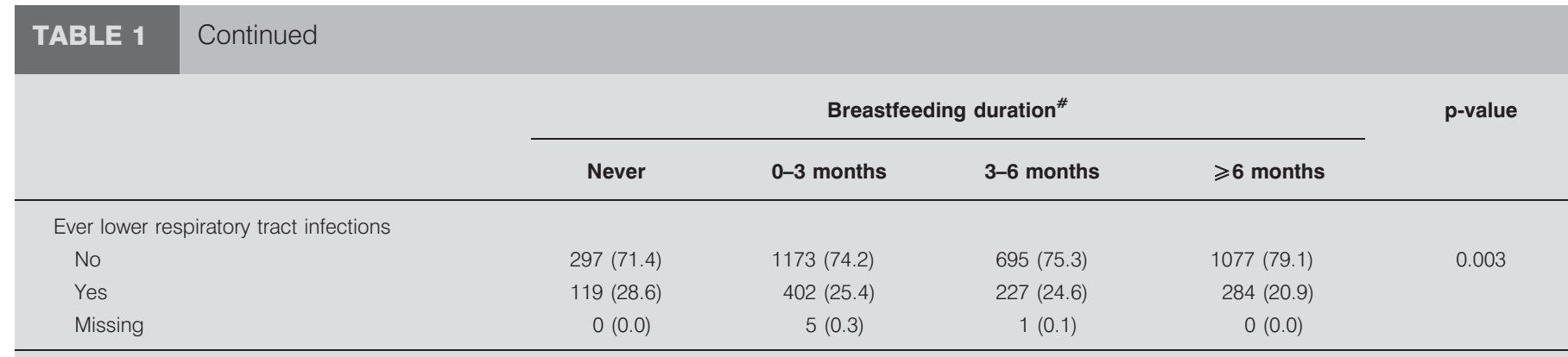

Data are presented as mean \pm SD or $n(\%)$, unless otherwise stated. Differences between breastfeeding groups were evaluated using Chi-squared tests for categorical values and one-way ANOVA for continues variables (only $p$-values between the never and $>6$ months breastfed groups are given). ${ }^{\#}: n=4,280$

\section{RESULTS}

Out of the total group of 5,368 children, $92.3 \%$ had ever been breastfed. Of those, information about duration and exclusiveness of breastfeeding was available for $79.7 \%(n=4,280)$ and $81.1 \%(n=4,353)$ of children, respectively. The median duration of breastfeeding was 3.5 months (95\% range 0.5-12.0 months) and $21.3 \%$ were breastfed exclusively until the age of 4 months. Table 1 shows the parental and child characteristics according to breastfeeding duration. Wheezing was the most frequently reported asthma-related symptom during the first year (table 2). Child and parental characteristics differed between those with and without available data on asthma-related symptoms except for sex and ever eczema (online supplementary material table S1). The effect sizes of unadjusted and nonimputed analyses (online supplementary material tables S2 and S3) of the associations of duration and exclusiveness of breastfeeding with asthmarelated symptoms did not materially change after adjustment for confounders or performing multiple imputations of the confounders.

\section{Duration of breastfeeding}

Based on the GEE models, those who were never breastfed had overall increased risks of wheezing, shortness of breath, dry cough and persistent phlegm (OR 1.44 (95\% CI 1.24-1.66), 1.26 (1.07-1.48), 1.25 (1.08-1.44) and 1.57 (1.29-1.91), respectively) during the first $4 \mathrm{yrs}$, compared with children who were breastfed for $>6$ months (fig. 1). Analyses focused on these symptoms per year showed that children who had been breastfed for shorter periods had increased risks of wheezing at 1, 2 and 3 yrs $(p<0.05)$ (fig. 1a). A nonsignificant trend in the same direction was observed at the age of 4 yrs. Prolonged breastfeeding was associated with a lower risk of shortness of breath at 1 yr (1.38 (1.05-1.80)) (fig. 1b) and nonsignificant trends were observed for the older ages. Breastfeeding duration was also associated with the risk of dry cough at 3 yrs, but not at other ages (fig. 1c), and with the risk of persistent phlegm at 1, 3 and 4 yrs (fig. 1d). Effect estimates for each specific exposure and the asthma-related symptoms (dose-response) are given in the online supplementary material (table S4). Based on the discrete survival analysis, those who were never breastfed, or breastfed for 0-3 or 3-6 months tended to have asthma-related symptoms earlier in life compared with those who were breastfed for $>6$ months (hazard ratio (HR) 1.13 (95\% CI 0.97-1.32), 1.06 (0.961.17 ) and 1.03 (0.92-1.15), respectively) (fig. 2).

\section{Exclusiveness of breastfeeding}

Subjects who were nonexclusively breastfed for 4 months had increased risks of wheezing, shortness of breath, dry cough and persistent phlegm during the first 4 yrs (OR 1.21 (95\% CI 1.09$1.34), 1.14$ (1.02-1.28), 1.20 (1.10-1.31) and 1.21 (1.04-1.42), respectively) compared with children who were exclusively breastfed for 4 months (fig. 3). Analyses focused on each year separately showed that, compared with children who had been exclusively breastfed for 4 months, those who had been nonexclusively breastfed for 4 months had an increased risk of wheezing at 1, 2 and 3 yrs $(\mathrm{p}<0.05)$. Nonsignificant results were observed at 4 yrs. We observed similar but less consistent tendencies for dry cough (fig. 3c), but not for shortness of breath and persistent phlegm (fig. $3 b$ and $d$ ). Based on the discrete survival analysis, those who were never or not exclusively breastfed for 4 months had asthma-related symptoms earlier in

TABLE 2 Frequencies of asthma-related symptoms

\begin{tabular}{lcccc} 
& \multicolumn{4}{c}{ Age yrs } \\
\cline { 2 - 5 } & $\mathbf{1}$ & $\mathbf{2}$ & $\mathbf{3}$ & $\mathbf{4}$ \\
\hline Subjects & 4787 & 4644 & 4301 & 4297 \\
Wheezing & 4493 & 4551 & 4228 & 4219 \\
No & $3202(71.3)$ & $3638(79.9)$ & $3691(87.3)$ & $3675(87.1)$ \\
Yes & $1291(28.7)$ & $913(20.1)$ & $537(12.7)$ & $544(12.9)$ \\
$\quad 1-3$ times per year & $992(22.1)$ & $756(16.6)$ & $432(10.2)$ & $449(10.6)$ \\
$\quad \geqslant 4$ times per year & $299(6.7)$ & $157(3.4)$ & $105(2.5)$ & $95(2.3)$ \\
Shortness of breath & 4498 & 4570 & 4236 & 4239 \\
No & $3495(77.7)$ & $3750(82.1)$ & $3738(88.2)$ & $3787(89.3)$ \\
Yes & $1,003(22.3)$ & $820(17.9)$ & $498(11.8)$ & $452(10.7)$ \\
$\quad 1-3$ times per year & $781(17.4)$ & $642(14.0)$ & $396(9.3)$ & $346(8.2)$ \\
$\quad \geqslant 4$ times per year & $222(4.9)$ & $178(3.9)$ & $102(2.4)$ & $106(2.5)$ \\
Dry cough & 4446 & 4579 & 4191 & 4231 \\
$\quad$ No & $3453(77.7)$ & $3484(76.1)$ & $3200(76.4)$ & $3099(73.2)$ \\
Yes & $993(22.3)$ & $1095(23.9)$ & $991(23.6)$ & $1132(26.8)$ \\
Persistent phlegm & 4437 & 4541 & 4267 & 4267 \\
No & $3854(86.9)$ & $4098(90.2)$ & $3986(93.4)$ & $3959(92.8)$ \\
Yes & $583(13.1)$ & $443(9.8)$ & $281(6.6)$ & $308(7.2)$ \\
\hline
\end{tabular}

Data are presented as $\mathrm{n}$ or $\mathrm{n}(\%)$ 


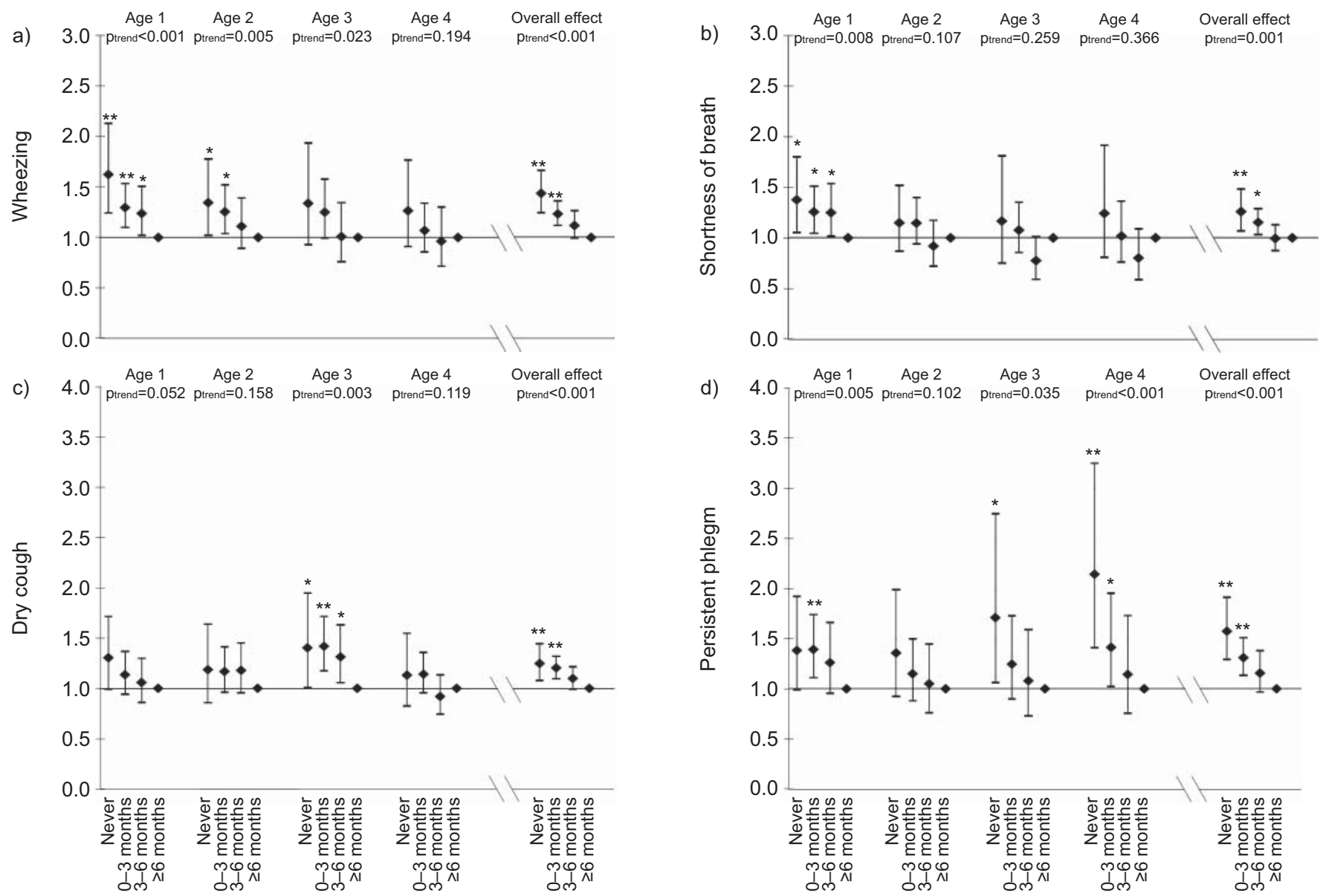

FIGURE 1. Associations of breastfeeding duration with a) wheezing, b) shortness of breath, c) dry cough and d) persistent phlegm until the age of 4 yrs. Data are presented as odds ratios (ORs) with 95\% confidence intervals from longitudinal generalised estimating equation models. ORs are given for the overall effect and (allowing for a time trend) for each year of age separately. Children who were breastfed for $>6$ months were used as a reference category. Models are adjusted for parental age, education, ethnicity, smoking habits, maternal parity, children's sex, gestational age, birth weight, parental history of asthma or atopy, daycare attendance and pet keeping. Ptrend: p-value for trend. $*: p<0.05 ; * *: p<0.01$

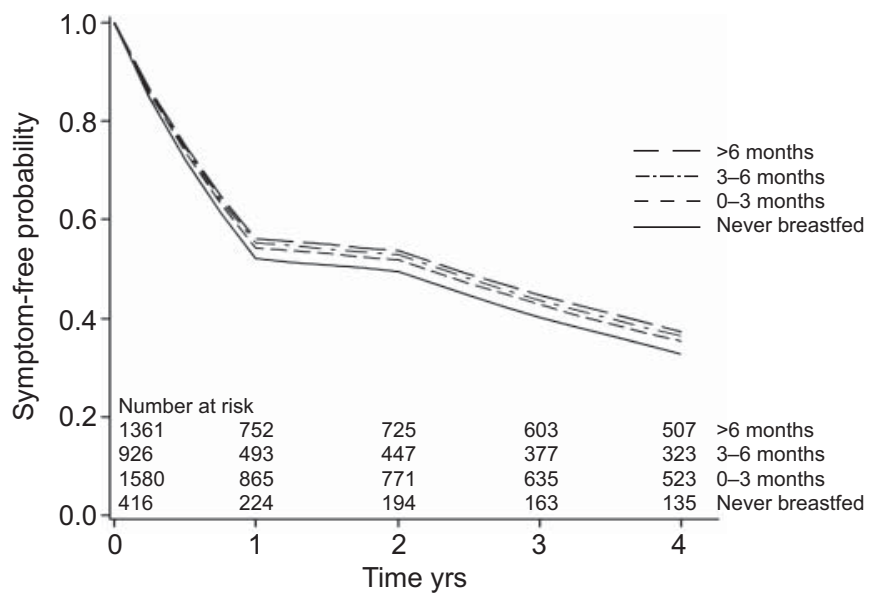

FIGURE 2. Time to any first asthma-related symptom (discrete survival curves) according to duration of breastfeeding. Models are adjusted for parental age, education, ethnicity, smoking habits, maternal parity, children's sex, gestational age, birth weight, parental history of asthma or atopy, daycare attendance and pet keeping by taking the mean of the values. life compared with those who were exclusively breastfed (HR 1.23 (95\% CI 1.05-1.44) and 1.14 (1.03-1.26), respectively) (fig. 4).

\section{Atopy and infections}

Adjustment for eczema did not materially change the effect estimates of the association between breastfeeding exclusiveness with asthma-related symptoms in the first 4 yrs of life, whereas the estimates decreased when lower respiratory tract infections were added as a confounder (fig. 5 and online supplementary table S6).

\section{Effect modification analyses}

Differences in the overall risk of wheezing were observed for nonexclusively breastfed children with and without a parental history of asthma or atopy (OR 1.27 (1.11-1.45) and 1.14 (0.961.35), respectively) (online supplementary material, fig. S2 and table S7). However, no effect modification by parental history of asthma or atopy was observed for the associations of exclusiveness of breastfeeding with wheezing ( $p$-values interaction term $>0.05$ in the GEE model).

\section{DISCUSSION}

Shorter duration and nonexclusivity of breastfeeding were associated with increased risks of asthma-related symptoms in 

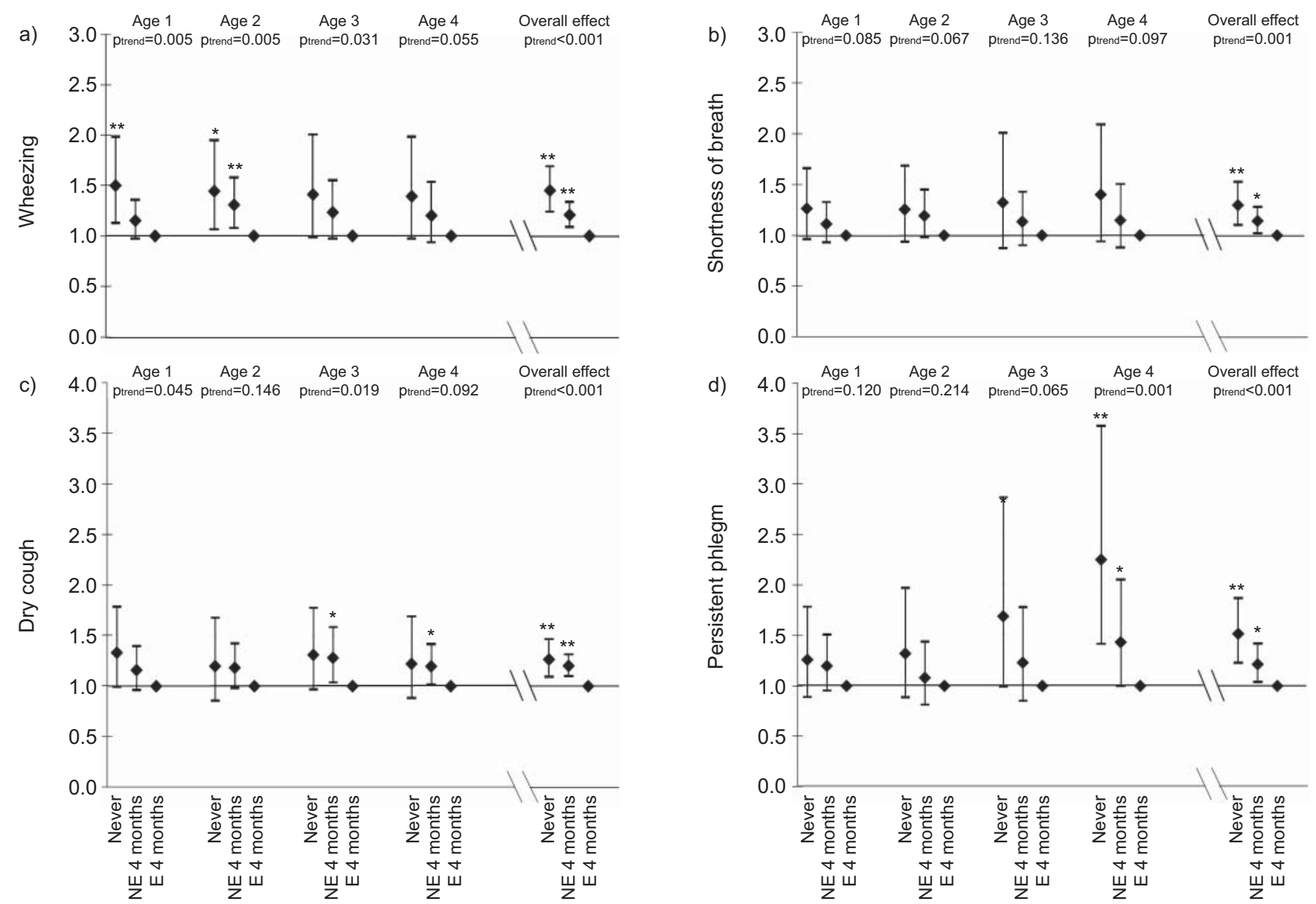

FIGURE 3. Associations of exclusive breastfeeding with a) wheezing, b) shortness of breath, c) dry cough and d) persistent phlegm until the age of 4 yrs. Data are presented as odds ratios (ORs) with 95\% confidence intervals from longitudinal generalised estimating equation models. ORs are given for the overall effect and (allowing for a time trend) for each year of age separately. Children who were exclusively breastfed for 4 months were used as reference category. Models are adjusted for parental age, education, ethnicity, smoking habits, maternal parity, children's sex, gestational age, birth weight, parental history of asthma or atopy, daycare attendance and pet keeping NE: nonexclusive; E: exclusive; ptrend: $p$-value for trend. ${ }^{*}: p<0.05 ; * *: p<0.01$.

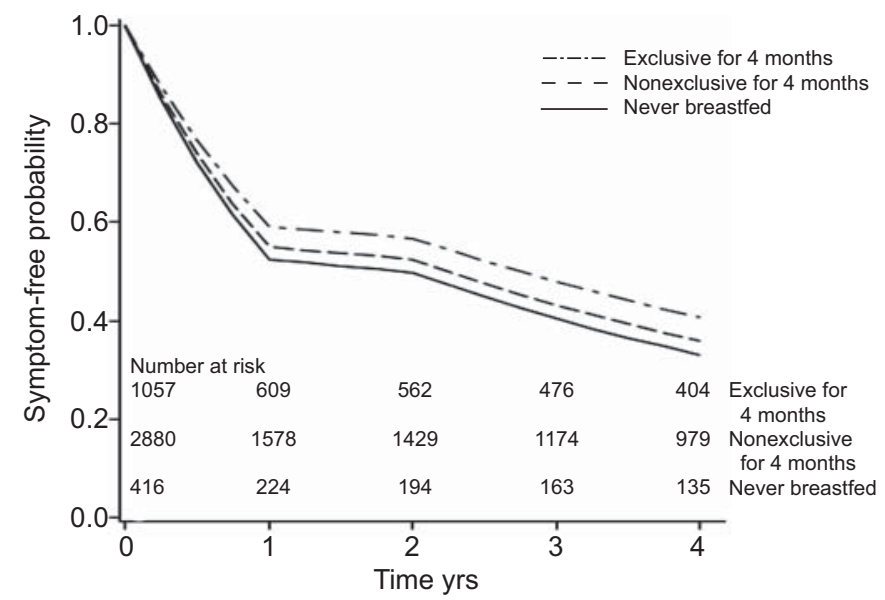

FIGURE 4. Time to any first asthma-related symptom (discrete survival curves) according to exclusivity of breastfeeding. Models are adjusted for parental age, education, ethnicity, smoking habits, maternal parity, children's sex, gestational age, birth weight, parental history of asthma or atopy, daycare attendance and pet keeping by taking the mean of the values. preschool children. The strongest effect estimates were observed for wheezing during the first 2 yrs.

Previous studies reported consistent results on the associations between duration and exclusive breastfeeding and the risk of asthma in childhood. These suggested an up to 2.22-fold increased risk of recurrent wheezing or asthma at the ages of 26 yrs among children who were not breastfed or not exclusively breastfed until the age of 4 months [3-11,13]. Our effect estimates are in line with these studies, and, additionally, we observed a dose-response relationship between breastfeeding and the number of wheezing episodes. We observed similar results for shortness of breath, dry cough at night and persistent phlegm. Also, we found that the first reported asthma-related symptom occurred earlier in life if children were either breastfed for a shorter period of time or were nonexclusively breastfed. We found evidence for a protective effect of breastfeeding on wheezing until the age of $2 \mathrm{yrs}$, but not thereafter. In these first years, wheezing is predominantly associated with respiratory tract infections [22]. Indeed, we observed that the protective effect of breastfeeding on asthma-related symptoms decreased 

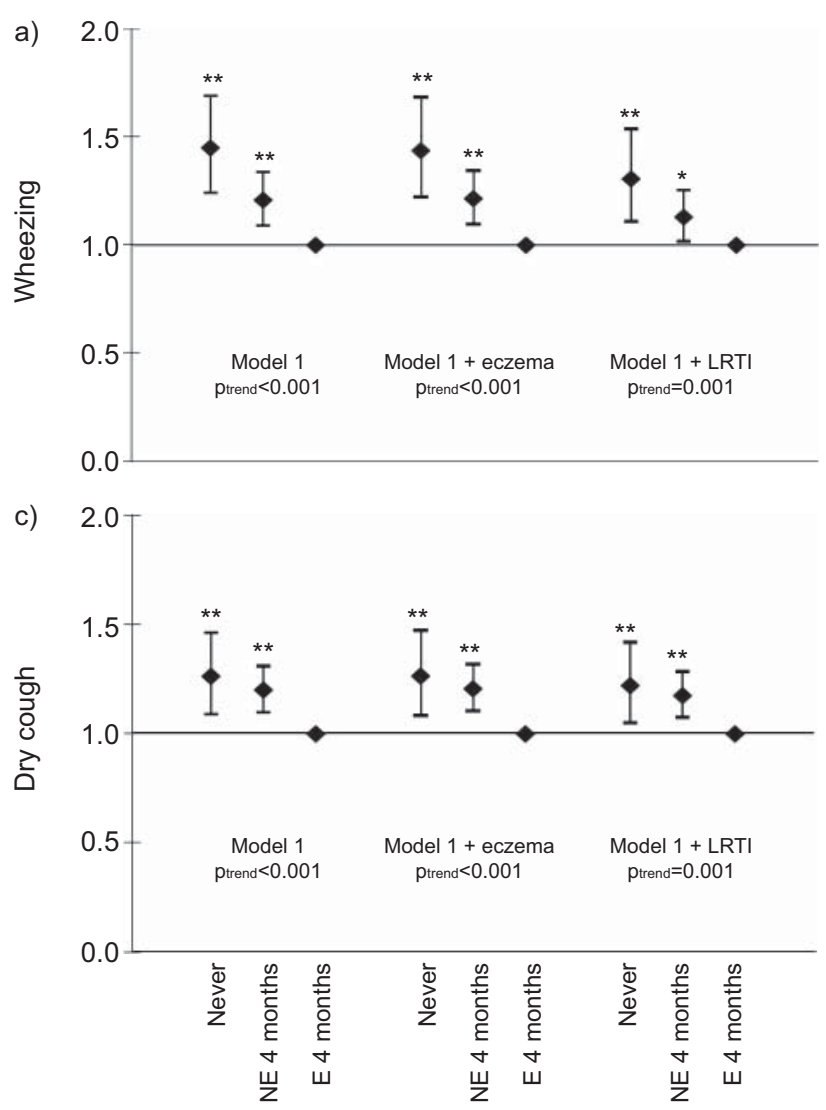
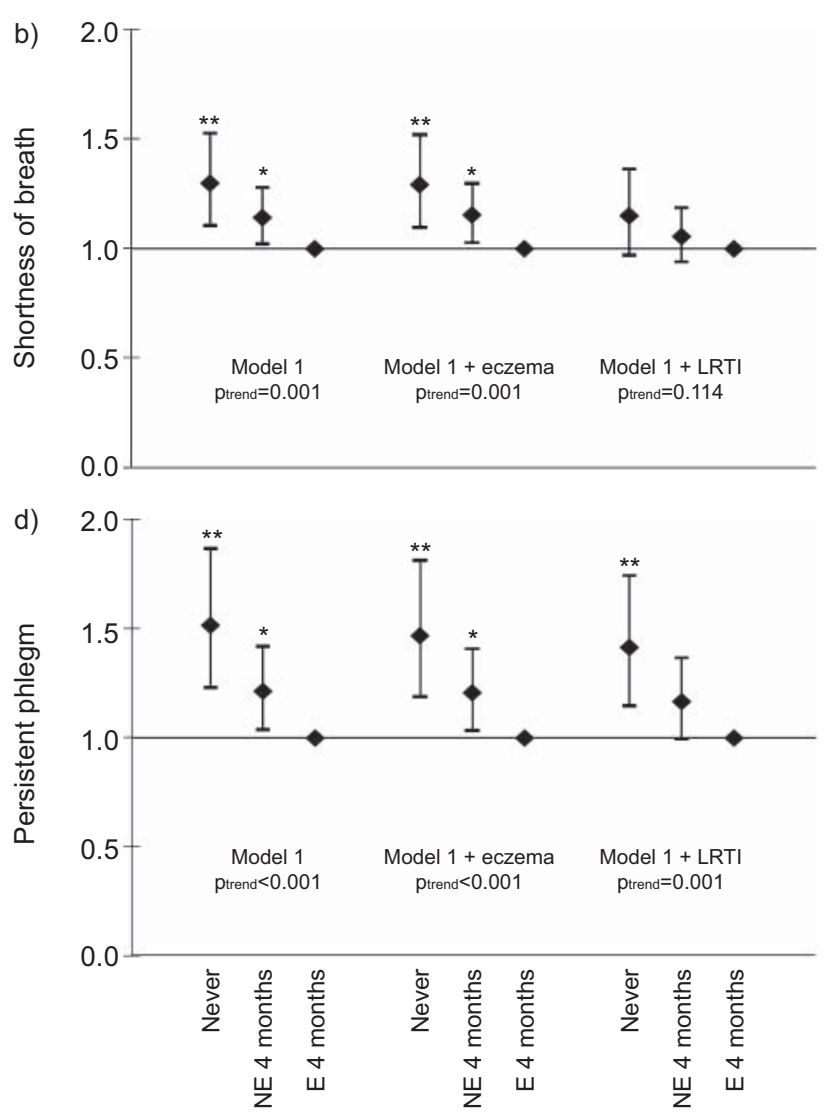

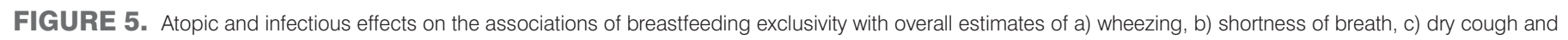

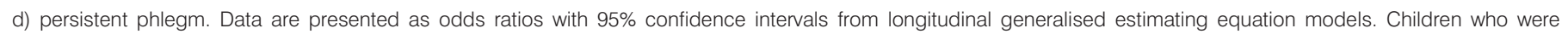

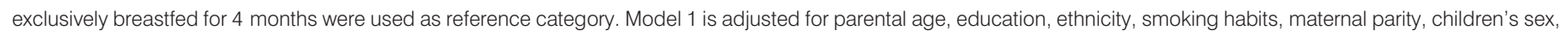

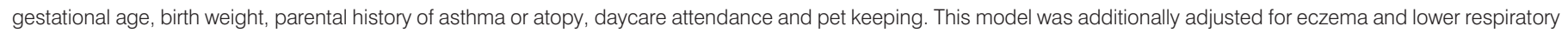
tract infections (LRTI), which were both not imputed. NE: nonexclusive; $E$ : exclusive; ptrend: $p$-value for trend. ${ }^{*}: p<0.05$; ${ }^{\star} *$ : $p<0.01$.

after adjusting for lower respiratory tract infections at the corresponding ages.

The gut microflora is suggested to be different between breastfed and formula-fed infants. Compared with breastfed infants, those who receive formula have a more complex microflora with facultative anaerobes, bacteroides and clostridia at higher levels and frequencies [23]. We speculate that this might decrease with increasing exclusivity of breastfeeding, leading to a lower infection risk and less wheezing by influencing the development of the immune system [22]. Due to this putative effect on the development of the immune system, infections and asthmarelated symptoms might occur less frequently even years after stopping breastfeeding. This is in line with the previously reported inconsistent results for the association of breastfeeding with the risk of asthma after the preschool age, as in that period, the gut microflora has stabilised, respiratory tract infections are less frequent and atopic mechanisms are more relevant. Also, our results regarding the nonsignificant associations with asthmarelated symptoms at older ages are in line with a previously published randomised clustered trial [24].

Previous studies reported inconclusive or inversed associations of breastfeeding with eczema or atopy. Also, breastfeeding is suggested to have a potential adverse long-term effect on asthma, which demonstrates the difficulties of giving breastfeeding advice for atopy prevention [2, 5, 8]. We did not observe a change in effect estimates for asthma-related symptoms after adjusting for eczema, but only found significant effects of nonexclusive breastfeeding in children with a parental history of asthma or atopy, suggesting a larger effect of breastfeeding in this group. However, the interaction term was not significant, which may be due to the lack of large statistical power. Our results suggest that the associations of breastfeeding exclusiveness with asthma-related symptoms are at least partly modified by parental asthma or atopy. Previously, WrigHT et al. [8] also observed different relationships between breastfeeding and asthma with the presence or absence of maternal asthma and atopy. Breastfed children of asthmatic mothers had an increased risk of asthma from the age of 6 yrs onwards compared with breastfed children of nonasthmatic mothers. However, other studies did not report effect modification of a parental history of asthma or atopy on the association of breastfeeding with wheezing $[5,11,12]$.

This study was embedded in a population-based prospective design with a large number of subjects being studied from early life onwards, and information about a large number of potential confounders was prospectively collected. We adjusted for a large number of confounders and the results did not differ between 
nonimputed and imputed analysis. However, we cannot exclude that other possible (residual) confounders, effect modifiers or the influence of genetic variances might have been present.

Nonresponse would lead to biased effect estimates if the associations of breastfeeding duration and exclusivity with asthma-related symptoms were different between those included and not included in the analyses. However, this seems unlikely because biased estimates mainly arose from loss to follow-up rather than from nonresponse at baseline [25].

Among infants without data on asthma-related symptoms, the frequencies of breastfeeding were lower than among infants with information on symptoms. This might have led to some loss of power and underestimation of the observed protective effects of breastfeeding in our cohort.

The main outcomes in our study were self-reported asthmarelated symptoms. This method is widely accepted in epidemiological studies and reliably reflects the incidence of asthma-related symptoms in young children [26]. In preschool children, a diagnosis of asthma is based on symptoms [27]. Objective tests, including lung function or bronchial hyperresponsiveness, are difficult to perform in young children or not informative. The most consistent protective effects of breastfeeding over time were observed for wheezing. For the other asthma-related symptoms, more varying and inconsistent patterns from birth to the age of 4 yrs were found. This might be due to lower prevalence of these symptoms and the possibility that these are related to infections rather than wheeze, representing other diseases more accurately, such as respiratory tract infections. Reversed causality might be present if the duration and exclusiveness of breastfeeding had been influenced by early manifestation of asthma-related symptoms and could have led to underestimation of the effect estimates $[5,7,8,15,28]$. In our cohort, we assessed only one asthma-related symptom, wheezing (no or yes), at $<2$ months of age $(n=4,130)$. Of children who wheezed in their first year $(n=1,291), 18.8 \%$ had already had a wheezing episode in the first 2 months. The frequencies of the duration and exclusiveness of breastfeeding were similar in those who had and had not had a first wheezing episode at the age of 2 months (duration of breastfeeding $>6$ months was $25.1 \%$ versus $26.5 \%$; exclusive breastfeeding $19.8 \%$ versus $18.2 \%$ ). Furthermore, when we additionally adjusted our presented analyses for wheezing before the age of 2 months, the effect estimates did not materially change. Therefore, it is unlikely that reversed causation was present in our cohort.

In conclusion, our results suggest that a short duration of breastfeeding and nonexclusivity are associated with increased risks of the asthma-related symptoms during the first 4 yrs of life, with the strongest effect estimates during the first 2 yrs. These associations seem to be partly explained by lower respiratory tract infections, but not by atopic mechanisms. Further studies are needed to explore the underlying mechanisms and the protective effect of breastfeeding on the various types of asthma in later life.

\section{SUPPORT STATEMENT}

The Generation R Study is made possible by financial support from the Erasmus Medical Center (Rotterdam, the Netherlands), the Erasmus University Rotterdam, the Netherlands Organization for Health Research and Development (ZonMw), the Netherlands Organisation for Scientific Research (NWO), the Ministry of Health, Welfare and Sport, and the Ministry of Youth and Families. L. Duijts received funding by means of a European Respiratory Society/Marie Curie Joint Research Fellowship (MC 1226-2009) under grant agreement RESPIRE, PCOFUND-GA-2008229571. V.W.V. Jaddoe received additional grants from the Netherlands Organization for Health Research and Development (ZonMw 90700303 and 916.10159).

\section{STATEMENT OF INTEREST}

None declared.

\section{ACKNOWLEDGEMENTS}

The Generation R Study is conducted by the Erasmus Medical Center in close collaboration with the School of Law and Faculty of Social Sciences of the Erasmus University Rotterdam, the Municipal Health Service Rotterdam area, the Rotterdam Homecare Foundation and the Stichting Trombosedienst and Artsenlaboratorium Rijnmond (STAR-MDC); all Rotterdam, the Netherlands. We gratefully acknowledge the contribution of children and parents, general practitioners, hospitals, midwives and pharmacies in Rotterdam. We thank D. Caudri (Dept of Paediatrics, Erasmus Medical Center, Rotterdam, the Netherlands) for his valuable comments on the statistical analyses.

\section{REFERENCES}

1 World Health Organization. The Global Burden of Disease - 2004 Update. Geneva, WHO, 2004.

2 Midodzi WK, Rowe BH, Majaesic CM, et al. Early life factors associated with incidence of physician-diagnosed asthma in preschool children: results from the Canadian Early Childhood Development cohort study. J Asthma 2010; 47: 7-13.

3 Fredriksson P, Jaakkola N, Jaakkola JJ. Breastfeeding and childhood asthma: a six-year population-based cohort study. BMC Pediatr 2007; 7: 39.

4 Giwercman C, Halkjaer LB, Jensen SM, et al. Increased risk of eczema but reduced risk of early wheezy disorder from exclusive breast-feeding in high-risk infants. J Allergy Clin Immunol 2010; 125: 866-871.

5 Elliott L, Henderson J, Northstone K, et al. Prospective study of breast-feeding in relation to wheeze, atopy, and bronchial hyperresponsiveness in the Avon Longitudinal Study of Parents and Children (ALSPAC). J Allergy Clin Immunol 2008; 122: 49-54.

6 Kull I, Almqvist C, Lilja G, et al. Breast-feeding reduces the risk of asthma during the first 4 years of life. J Allergy Clin Immunol 2004; 114: 755-760.

7 Kull I, Melen E, Alm J, et al. Breast-feeding in relation to asthma, lung function, and sensitization in young schoolchildren. J Allergy Clin Immunol 2010; 125: 1013-1019.

8 Wright AL, Holberg CJ, Taussig LM, et al. Factors influencing the relation of infant feeding to asthma and recurrent wheeze in childhood. Thorax 2001; 56: 192-197.

9 Oddy WH, Sly PD, de Klerk NH, et al. Breast feeding and respiratory morbidity in infancy: a birth cohort study. Arch Dis Child 2003; 88: 224-228.

10 Oddy WH, Holt PG, Sly PD, et al. Association between breast feeding and asthma in 6 year old children: findings of a prospective birth cohort study. BMJ 1999; 319: 815-819.

11 Scholtens S, Wijga AH, Brunekreef B, et al. Breast feeding, parental allergy and asthma in children followed for 8 years. The PIAMA birth cohort study. Thorax 2009; 64: 604-609.

12 Rothenbacher D, Weyermann M, Beermann C, et al. Breastfeeding, soluble CD14 concentration in breast milk and risk of atopic dermatitis and asthma in early childhood: birth cohort study. Clin Exp Allergy 2005; 35: 1014-1021. 
13 Snijders BE, Thijs C, Dagnelie PC, et al. Breast-feeding duration and infant atopic manifestations, by maternal allergic status, in the first 2 years of life (KOALA study). J Pediatr 2007; 151: 347-351.

14 Karmaus W, Dobai AL, Ogbuanu I, et al. Long-term effects of breastfeeding, maternal smoking during pregnancy, and recurrent lower respiratory tract infections on asthma in children. J Asthma 2008; 45: 688-695.

15 Sears MR, Greene JM, Willan AR, et al. Long-term relation between breastfeeding and development of atopy and asthma in children and young adults: a longitudinal study. Lancet 2002; 360: 901-907.

16 Mandhane PJ, Greene JM, Sears MR. Interactions between breastfeeding, specific parental atopy, and sex on development of asthma and atopy. J Allergy Clin Immunol 2007; 119: 1359-1366.

17 Friedman NJ, Zeiger RS. The role of breast-feeding in the development of allergies and asthma. J Allergy Clin Immunol 2005; 115: 1238-1248.

18 Newburg DS. Neonatal protection by an innate immune system of human milk consisting of oligosaccharides and glycans. J Anim Sci 2009; 87: 26-34.

19 Hoppu U, Kalliomaki M, Laiho K, et al. Breast milk - immunomodulatory signals against allergic diseases. Allergy 2001; 56: Suppl. 67, 23-26.

20 Jaddoe VW, van Duijn CM, van der Heijden AJ, et al. The Generation R Study: design and cohort update 2010. Eur J Epidemiol 2010; 25: 823-841.
21 Asher MI, Keil U, Anderson HR, et al. International Study of Asthma and Allergies in Childhood (ISAAC): rationale and methods. Eur Respir J 1995; 8: 483-491.

22 Friedlander SL, Jackson DJ, Gangnon RE, et al. Viral infections, cytokine dysregulation and the origins of childhood asthma and allergic diseases. Pediatr Infect Dis J 2005; 24: Suppl. 11, S170-S176.

23 Mountzouris KC, McCartney AL, Gibson GR. Intestinal microflora of human infants and current trends for its nutritional modulation. Br J Nutr 2002; 87: 405-420.

24 Kramer MS, Matush L, Vanilovich I, et al. Effect of prolonged and exclusive breast feeding on risk of allergy and asthma: cluster randomised trial. BMJ 2007; 335: 815.

25 Nohr EA, Frydenberg M, Henriksen TB, et al. Does low participation in cohort studies induce bias? Epidemiology 2006; 17: 413-418.

26 Jenkins MA, Clarke JR, Carlin JB, et al. Validation of questionnaire and bronchial hyperresponsiveness against respiratory physician assessment in the diagnosis of asthma. Int J Epidemiol 1996; 25: 609-616.

27 Edwards CA, Osman LM, Godden DJ, et al. Wheezy bronchitis in childhood: a distinct clinical entity with lifelong significance? Chest 2003; 124: 18-24.

28 Guilbert TW, Stern DA, Morgan WJ, et al. Effect of breastfeeding on lung function in childhood and modulation by maternal asthma and atopy. Am J Respir Crit Care Med 2007; 176: 843-848. 